\title{
THE SOLVABILITY AND SUBELLIPTICITY OF SYSTEMS OF PSEUDODIFFERENTIAL OPERATORS
}

\author{
NILS DENCKER \\ Dedicated to Ferruccio Colombini on his sixtieth birthday
}

\section{INTRODUCTION}

In this paper we shall study the question of solvability and subellipticity of square systems of classical pseudodifferential operators of principal type on a $C^{\infty}$ manifold $X$. These are the pseudodifferential operators which have an asymptotic expansion in homogeneous terms, where the highest order term, the principal symbol, vanishes of first order on the kernel. Local solvability for an $N \times N$ system of pseudodifferential operators $P$ at a compact set $K \subseteq X$ means that the equations

$$
P u=v
$$

have a local weak solution $u \in \mathcal{D}^{\prime}\left(X, \mathbf{C}^{N}\right)$ in a neighborhood of $K$ for all $v \in C^{\infty}\left(X, \mathbf{C}^{N}\right)$ in a subset of finite codimension. We can also define microlocal solvability at any compactly based cone $K \subset T^{*} X$, see [5, Definition 26.4.3]. Hans Lewy's famous counterexample [6] from 1957 showed that not all smooth linear partial differential operators are solvable.

In the scalar case, Nirenberg and Treves conjectured in [7] that local solvability of scalar classical pseudodifferential operators of principal type is equivalent to condition $(\Psi)$ on the principal symbol $p$. Condition $(\Psi)$ means that

(1.2) $\operatorname{Im}(a p)$ does not change sign from - to +

$$
\text { along the oriented bicharacteristics of } \operatorname{Re}(a p)
$$

for any $0 \neq a \in C^{\infty}\left(T^{*} X\right)$. These oriented bicharacteristics are the positive flow-outs of the Hamilton vector field

$$
H_{\operatorname{Re}(a p)}=\sum_{j} \partial_{\xi_{j}} \operatorname{Re}(a p) \partial_{x_{j}}-\partial_{x_{j}} \operatorname{Re}(a p) \partial_{\xi_{j}}
$$

on $\operatorname{Re}(a p)=0$, and are called semibicharacteristics of $p$. The Nirenberg-Treves conjecture was recently proved by the author, see [2].

Date: December 1, 2008.

2000 Mathematics Subject Classification. 35S05 (primary) 35A07, 35H20, 47G30, 58J40 (secondary).

Key words and phrases. solvability, subelliptic, pseudodifferential operator, principal type, systems. 
Condition (1.2) is obviously invariant under symplectic changes of coordinates and multiplication with non-vanishing factors. Thus the condition is invariant under conjugation of $P$ with elliptic Fourier integral operators. We say that $p$ satisfies condition $(\bar{\Psi})$ if $\bar{p}$ satisfies condition $(\Psi)$, which means that only sign changes from - to + is allowed in (1.2). We also say that $p$ satisfies condition $(P)$ if there are no sign changes on the semibicharacteristics, that is, $p$ satisfies both condition $(\Psi)$ and $(\bar{\Psi})$. For partial differential operators condition $(\Psi)$ and $(P)$ are equivalent, since the principal symbol is either odd or even in $\xi$.

For systems there is no corresponding conjecture for solvability. We shall consider systems of principal type, so that the principal symbol vanishes of first order on the kernel, see Definition 2.1. By looking at diagonal operators, one finds that condition $(\Psi)$ for the eigenvalues of the principal symbol is necessary for solvability. A special case is when we have constant characteristics, so that the eigenvalue close to the origin has constant multiplicity, see Definition 2.6. Then, the eigenvalue is a $C^{\infty}$ function and condition $(\Psi)$ is well-defined. For classical systems of pseudodifferential operators of principal type having eigenvalues of the principal symbol with constant multiplicity, the generalization of the Nirenberg-Treves conjecture is that local solvability is equivalent to condition $(\Psi)$ on the eigenvalues. This has recently been proved by the author, see Theorem 2.7 in [4].

But when the principal symbol is not diagonalizable, condition $(\Psi)$ is not sufficient for local solvability, see Example 2.7 below. In fact, it is not even known if condition $(\Psi)$ is sufficient in the case when the principal system is $C^{\infty}$ diagonalizable. Instead, we shall study the quasi-symmetrizable systems introduced in [3], see Definition 2.8. These are of principal type, are invariant under taking adjoints and multiplication with invertible systems. A scalar quasi-symmetrizable symbol is of principal type and satisfies condition $(P)$. Our main result is that quasi-symmetrizable systems are locally solvable, see Theorem 2.17.

We shall also study the subellipticity of square systems. An $N \times N$ system of pseudodifferential operators $P \in \Psi_{c l}^{m}(X)$ is subelliptic with a loss of $\gamma<1$ derivatives if $P u \in H_{(s)}$ implies that $u \in H_{(s+m-\gamma)}$ locally for $u \in \mathcal{D}^{\prime}\left(X, \mathbf{C}^{N}\right)$. Here $H_{(s)}$ are the standard $L^{2}$ Sobolev spaces, thus ellipticity corresponds to $\gamma=0$ so we may assume $\gamma>0$. For scalar operators, subellipticity is equivalent to condition $(\bar{\Psi})$ and the bracket condition on the principal symbol $p$, i.e., that some repeated Poisson bracket of $\operatorname{Re} p$ and $\operatorname{Im} p$ is non-vanishing. This is not true for systems, and there seems to be no general results on the subellipticity for systems of pseudodifferential operators. In fact, the real and imaginary parts do not commute in general, making the bracket condition meaningless. Even 
when they do, the bracket condition is not invariant and not sufficient for subellipticity, see Example 3.2.

Instead we shall study quasi-symmetrizable symbols, for which we introduce invariant conditions on the order of vanishing of the symbol along the semibicharacteristics of the eigenvalues. Observe that for systems, there could be several (limit) semibicharacteristics of the eigenvalues going through a characteristic point, see Example 3.10. Therefore we introduce the approximation property in Definition 3.11 which gives that the all (limit) semibicharacteristics of the eigenvalues are parallell at the characteristics, see Remark 3.12. We shall study systems of finite type introduced in [3], these are quasisymmetrizable systems satisfying the approximation property, for which the imaginary part on the kernel vanishes of finite order along the bicharacteristics of the real part of the eigenvalues. This definition is invariant under multiplication with invertible systems and taking adjoints. For scalar symbols this corresponds to the case when the operator satisfies condition $(P)$ and the bracket condition. For system of finite type we obtain subellipticity with a loss of $2 k / 2 k+1$ derivatives as in the scalar case, where $2 k$ is the order of vanishing, see Theorem 3.21. For the proof, we shall use the estimates developed in [3]. The results in this paper are formulated for operators acting on the trivial bundle. But since our results are mainly local, they can be applied to operators on sections of fiber bundles.

\section{Solvability of Systems}

Recall that a scalar symbol $p(x, \xi) \in C^{\infty}\left(T^{*} X\right)$ is of principal type if $d p \neq 0$ when $p=0$. We shall generalize this definition to systems $P \in C^{\infty}\left(T^{*} X\right)$. For $\nu \in T_{w}\left(T^{*} X\right)$, $w=(x, \xi)$, we let $\partial_{\nu} P(w)=\langle\nu, d P(w)\rangle$. We shall denote $\operatorname{Ker} P$ the kernel and Ran $P$ the range of the matrix $P$.

Definition 2.1. The $N \times N$ system $P(w) \in C^{\infty}\left(T^{*} X\right)$ is of principal type at $w_{0}$ if

$$
\operatorname{Ker} P\left(w_{0}\right) \ni u \mapsto \partial_{\nu} P\left(w_{0}\right) u \in \operatorname{Coker} P\left(w_{0}\right)=\mathbf{C}^{N} / \operatorname{Ran} P\left(w_{0}\right)
$$

is bijective for some $\nu \in T_{w_{0}}\left(T^{*} X\right)$. The operator $P \in \Psi_{c l}^{m}(X)$ is of principal type if the homogeneous principal symbol $\sigma(P)$ is of principal type.

Observe that if $P$ is homogeneous in $\xi$, then the direction $\nu$ cannot be radial. In fact, if $\nu$ has the radial direction and $P$ is homogeneous then $\partial_{\nu} P=c P$ which vanishes on Ker $P$.

Remark 2.2. If $P(w) \in C^{\infty}$ is of principal type and $A(w), B(w) \in C^{\infty}$ are invertible then $A P B$ is of principal type. We have that $P$ is of principal type if and only if the adjoint $P^{*}$ is of principal type. 
In fact, by Leibniz' rule we have

$$
\partial(A P B)=(\partial A) P B+A(\partial P) B+A P \partial B
$$

and $\operatorname{Ran}(A P B)=A(\operatorname{Ran} P)$ and $\operatorname{Ker}(A P B)=B^{-1}(\operatorname{Ker} P)$ when $A$ and $B$ are invertible, which gives invariance under left and right multiplication. Since $\operatorname{Ker} P^{*}\left(w_{0}\right)=$ Ran $P\left(w_{0}\right)^{\perp}$ we find that $P$ satisfies (2.1) if and only if

$$
\operatorname{Ker} P\left(w_{0}\right) \times \operatorname{Ker} P^{*}\left(w_{0}\right) \ni(u, v) \mapsto\left\langle\partial_{\nu} P\left(w_{0}\right) u, v\right\rangle
$$

is a non-degenerate bilinear form. Since $\left\langle\partial_{\nu} P^{*} v, u\right\rangle=\overline{\left\langle\partial_{\nu} P u, v\right\rangle}$ we then obtain that $P^{*}$ is of principal type.

Observe that if $P$ only has one vanishing eigenvalue $\lambda$ (with multiplicity one) then the condition that $P$ is of principal type reduces to the condition in the scalar case: $d \lambda \neq 0$ when $\lambda=0$. In fact, by using the spectral projection one can find invertible systems $A$ and $B$ so that

$$
A P B=\left(\begin{array}{ll}
\lambda & 0 \\
0 & E
\end{array}\right) \in C^{\infty}
$$

where $E$ is an invertible $(N-1) \times(N-1)$ system. Since this system is of principal type we obtain the result by the invariance.

Example 2.3. Consider the system

$$
P(w)=\left(\begin{array}{cc}
\lambda_{1}(w) & 1 \\
0 & \lambda_{2}(w)
\end{array}\right)
$$

where $\lambda_{j}(w) \in C^{\infty}, j=1,2$. Then $P(w)$ is not of principal type when $\lambda_{1}(w)=\lambda_{2}(w)=0$ since then $\operatorname{Ker} P(w)=\operatorname{Ran} P(w)=\mathbf{C} \times\{0\}$, which is preserved by $\partial P$.

Observe that the property of being of principal type is not stable under $C^{1}$ perturbation, not even when $P=P^{*}$ is symmetric by the following example.

Example 2.4. The system

$$
P(w)=\left(\begin{array}{cc}
w_{1}-w_{2} & w_{2} \\
w_{2} & -w_{1}-w_{2}
\end{array}\right)=P^{*}(w) \quad w=\left(w_{1}, w_{2}\right)
$$

is of principal type when $w_{1}=w_{2}=0$, but not of principal type when $w_{2} \neq 0$ and $w_{1}=0$. In fact,

$$
\partial_{w_{1}} P=\left(\begin{array}{cc}
1 & 0 \\
0 & -1
\end{array}\right)
$$

is invertible, and when $w_{2} \neq 0$ we have that

$$
\operatorname{Ker} P\left(0, w_{2}\right)=\operatorname{Ker} \partial_{w_{2}} P\left(0, w_{2}\right)=\{z(1,1): z \in \mathbf{C}\}
$$

which is mapped to $\operatorname{Ran} P\left(0, w_{2}\right)=\{z(1,-1): z \in \mathbf{C}\}$ by $\partial_{w_{1}} P$. The eigenvalues of $P(w)$ are $-w_{2} \pm \sqrt{w_{1}^{2}+w_{2}^{2}}$ which are equal if and only if $w_{1}=w_{2}=0$. When $w_{2} \neq 0$ the eigenvalue close to zero is $w_{1}^{2} / 2 w_{2}+\mathcal{O}\left(w_{1}^{4}\right)$ which has vanishing differential at $w_{1}=0$. 
Recall that the multiplicity of $\lambda$ as a root of the characteristic equation $\left|P(w)-\lambda \operatorname{Id}_{N}\right|=$ 0 is the algebraic multiplicity of the eigenvalue, and the dimension of $\operatorname{Ker}\left(P(w)-\lambda \operatorname{Id}_{N}\right)$ is the geometric multiplicity. Observe the geometric multiplicity is lower or equal to the algebraic, and for symmetric systems they are equal.

Remark 2.5. If the eigenvalue $\lambda(w)$ has constant algebraic multiplicity then it is a $C^{\infty}$ function.

In fact, if $k$ is the multiplicity then $\lambda=\lambda(w)$ solves $\partial_{\lambda}^{k-1}\left|P(w)-\lambda \operatorname{Id}_{N}\right|=0$ so we obtain this from the Implicit Function Theorem. This is not true when we have constant geometric multiplicity, for example $P(t)=\left(\begin{array}{ll}0 & 1 \\ t & 0\end{array}\right), t \in \mathbf{R}$, has geometric multiplicity equal to one for the eigenvalues $\pm \sqrt{t}$.

Observe that if the matrix $P(w)$ depend continuously on a parameter $w$, then the eigenvalues $\lambda(w)$ also depend continuously on $w$. Such a continuous function $\lambda(w)$ of eigenvalues we will call a section of eigenvalues of $P(w)$.

Definition 2.6. The $N \times N$ system $P(w) \in C^{\infty}$ has constant characteristics near $w_{0}$ if there exists an $\varepsilon>0$ such that any section of eigenvalues $\lambda(w)$ of $P(w)$ with $|\lambda(w)|<\varepsilon$ has both constant algebraic and constant geometric multiplicity in a neighborhood of $w_{0}$.

If $P$ has constant characteristics then the section of eigenvalues close to zero has constant algebraic multiplicity, thus it is a $C^{\infty}$ function close to zero. We obtain from Proposition 2.10 in [4] that if $P(w) \in C^{\infty}$ is an $N \times N$ system of constant characteristics near $w_{0}$, then $P(w)$ is of principal type at $w_{0}$ if and only if the algebraic and geometric multiplicities of $P$ agree at $w_{0}$ and $d \lambda\left(w_{0}\right) \neq 0$ for the $C^{\infty}$ section of eigenvalues $\lambda(w)$ for $P$ satisfying $\lambda\left(w_{0}\right)=0$, thus there are no non-trivial Jordan boxes in the normal form.

For classical systems of pseudodifferential operators of principal type and constant characteristics, the eigenvalues are homogeneous $C^{\infty}$ functions when the values are close to zero, so the condition $(\Psi)$ given by (1.2) is well-defined on the eigenvalues. Then, the natural generalization of the Nirenberg-Treves conjecture is that local solvability is equivalent to condition $(\Psi)$ on the eigenvalues. This has recently been proved by the author, see Theorem 2.7 in [4].

When the multiplicity of the eigenvalues of the principal symbol is not constant the situation is much more complicated. The following example shows that then it is not sufficient to have conditions only on the eigenvalues in order to obtain solvability, not even in the principal type case. 
Example 2.7. Let $x \in \mathbf{R}^{2}, D_{x}=\frac{1}{i} \partial_{x}$ and

$$
P\left(x, D_{x}\right)=\left(\begin{array}{cc}
D_{x_{1}} & x_{1} D_{x_{2}} \\
x_{1} D_{x_{2}} & -D_{x_{1}}
\end{array}\right)=P^{*}\left(x, D_{x}\right)
$$

This system is symmetric of principal type and $\sigma(P)$ has real eigenvalues $\pm \sqrt{\xi_{1}^{2}+x_{1}^{2} \xi_{2}^{2}}$ but

$$
\frac{1}{2}\left(\begin{array}{cc}
1 & -i \\
1 & i
\end{array}\right) P\left(\begin{array}{cc}
1 & 1 \\
-i & i
\end{array}\right)=\left(\begin{array}{cc}
D_{x_{1}}-i x_{1} D_{x_{2}} & 0 \\
0 & D_{x_{1}}+i x_{1} D_{x_{2}}
\end{array}\right)
$$

which is not solvable at $(0,0)$ because condition $(\Psi)$ is not satisfied. The eigenvalues of the principal symbol are now $\xi_{1} \pm i x_{1} \xi_{2}$.

Of course, the problem is that the eigenvalues are not invariant under multiplication with elliptic systems. We shall instead study quasi-symmetrizable systems, which generalize the normal forms of the scalar symbol at the boundary of the numerical range of the principal symbol, see Example 2.9.

Definition 2.8. The $N \times N$ system $P(w) \in C^{\infty}\left(T^{*} X\right)$ is quasi-symmetrizable with respect to a real $C^{\infty}$ vector field $V$ in $\Omega \subseteq T^{*} X$ if $\exists N \times N$ system $M(w) \in C^{\infty}\left(T^{*} X\right)$ so that

$$
\begin{aligned}
& \operatorname{Re}\langle M(V P) u, u\rangle \geq c\|u\|^{2}-C\|P u\|^{2} \quad c>0 \quad \forall u \in \mathbf{C}^{N} \\
& \operatorname{Im}\langle M P u, u\rangle \geq-C\|P u\|^{2} \quad \forall u \in \mathbf{C}^{N}
\end{aligned}
$$

on $\Omega$, the system $M$ is called a symmetrizer for $P$. If $P \in \Psi_{c l}^{m}(X)$ then it is quasisymmetrizable if the homogeneous principal symbol $\sigma(P)$ is quasi-symmetrizable when $|\xi|=1$, one can then choose a homogeneous symmetrizer $M$.

The definition is clearly independent of the choice of coordinates in $T^{*} X$ and choice of basis in $\mathbf{C}^{N}$. When $P$ is elliptic, we find that $P$ is quasi-symmetrizable with respect to any vector field since $\|P u\| \cong\|u\|$. Observe that the set of symmetrizers $M$ satisfying (2.4)(2.5) is a convex cone, a sum of two multipliers is also a multiplier. Thus for a given vector field $V$ it suffices to make a local choice of symmetrizer and then use a partition of unity to get a global one.

Example 2.9. A scalar function $p \in C^{\infty}$ is quasi-symmetrizable if and only

$$
p(w)=e(w)\left(w_{1}+i f\left(w^{\prime}\right)\right) \quad w=\left(w_{1}, w^{\prime}\right)
$$

for some choice of coordinates, where $f \geq 0$. Then 0 is at the boundary of the numerical range of $p$.

In fact, it is obvious that $p$ in (2.6) is quasi-symmetrizable. On the other hand, if $p$ is quasi-symmetrizable then there exists $m \in C^{\infty}$ such that $m p=p_{1}+i p_{2}$ where $p_{j}$ are real satisfying $\partial_{\nu} p_{1}>0$ and $p_{2} \geq 0$. Thus 0 is at the boundary of the numerical range 
of $p$. By using Malgrange preparation theorem and changing coordinates as in the proof of Lemma 4.1 in [1], we obtain the normal form (2.6) with $\pm f \geq 0$.

Taylor has studied symmetrizable systems of the type $D_{t} \mathrm{Id}+i K$, for which there exists $R>0$ making $R K$ symmetric (see Definition 4.3.2 in [8]). These systems are quasisymmetrizable with respect to $\partial_{\tau}$ with symmetrizer $R$. We shall denote $\operatorname{Re} A=\frac{1}{2}\left(A+A^{*}\right)$ and $i \operatorname{Im} A=\frac{1}{2}\left(A-A^{*}\right)$ the symmetric and antisymmetric parts of the matrix $A$. Next, we recall the following result from Proposition 4.7 in [3].

Remark 2.10. If the $N \times N$ system $P(w) \in C^{\infty}$ is quasi-symmetrizable then it is of principal type. Also, the symmetrizer $M$ is invertible if $\operatorname{Im} M P \geq c P^{*} P$ for some $c>0$.

Observe that by adding $i \varrho P^{*}$ to $M$ we may assume that $Q=M P$ satisfies

$$
\operatorname{Im} Q \geq(\varrho-C) P^{*} P \geq P^{*} P \geq c Q^{*} Q \quad c>0
$$

for $\varrho \geq C+1$, and then the symmetrizer is invertible by Remark 2.10 .

Remark 2.11. The system $P \in C^{\infty}$ is quasi-symmetrizable with respect to $V$ if and only if there exists an invertible symmetrizer $M$ such that $Q=M P$ satisfies

$$
\begin{aligned}
& \operatorname{Re}\langle(V Q) u, u\rangle \geq c\|u\|^{2}-C\|Q u\|^{2} \quad c>0 \\
& \operatorname{Im}\langle Q u, u\rangle \geq 0
\end{aligned}
$$

for any $u \in \mathbf{C}^{N}$.

In fact, by the Cauchy-Schwarz inequality we find

$$
|\langle(V M) P u, u\rangle| \leq \varepsilon\|u\|^{2}+C_{\varepsilon}\|P u\|^{2} \quad \forall \varepsilon>0 \quad \forall u \in \mathbf{C}^{N}
$$

Since $M$ is invertible, we also have that $\|P u\| \cong\|Q u\|$.

Definition 2.12. If $Q \in C^{\infty}\left(T^{*} X\right)$ satisfies (2.8)-(2.9) then $Q$ is quasi-symmetric with respect to the real $C^{\infty}$ vector field $V$.

The invariance properties of quasi-symmetrizable systems is partly due to the following properties of semibounded matrices. Let $U+V=\{u+v: u \in U \wedge v \in V\}$ for linear subspaces $U$ and $V$ of $\mathbf{C}^{N}$.

Lemma 2.13. Assume that $Q$ is an $N \times N$ matrix such that $\operatorname{Im} z Q \geq 0$ for some $0 \neq$ $z \in \mathbf{C}$. Then we find

$$
\operatorname{Ker} Q=\operatorname{Ker} Q^{*}=\operatorname{Ker}(\operatorname{Re} Q) \bigcap \operatorname{Ker}(\operatorname{Im} Q)
$$

and $\operatorname{Ran} Q=\operatorname{Ran}(\operatorname{Re} Q)+\operatorname{Ran}(\operatorname{Im} Q) \perp \operatorname{Ker} Q$. 
Proof. By multiplying with $z$ we may assume that $\operatorname{Im} Q \geq 0$, clearly the conclusions are invariant under multiplication with complex numbers. If $u \in \operatorname{Ker} Q$, then we have $\langle\operatorname{Im} Q u, u\rangle=\operatorname{Im}\langle Q u, u\rangle=0$. By using the Cauchy-Schwarz inequality on $\operatorname{Im} Q \geq 0$ we find that $\langle\operatorname{Im} Q u, v\rangle=0$ for any $v$. Thus $u \in \operatorname{Ker}(\operatorname{Im} Q)$ so $\operatorname{Ker} Q \subseteq \operatorname{Ker} Q^{*}$. We get equality and (2.10) by the rank theorem, since $\operatorname{Ker} Q^{*}=\operatorname{Ran} Q^{\perp}$.

For the last statement we observe that $\operatorname{Ran} Q \subseteq \operatorname{Ran}(\operatorname{Re} Q)+\operatorname{Ran}(\operatorname{Im} Q)=(\operatorname{Ker} Q)^{\perp}$ by (2.10) where we also get equality by the rank theorem.

Proposition 2.14. If $Q \in C^{\infty}\left(T^{*} X\right)$ is quasi-symmetric and $E \in C^{\infty}\left(T^{*} X\right)$ is invertible, then $E^{*} Q E$ and $-Q^{*}$ are quasi-symmetric.

Proof. First we note that (2.8) holds if and only if

$$
\operatorname{Re}\langle(V Q) u, u\rangle \geq c\|u\|^{2} \quad \forall u \in \operatorname{Ker} Q
$$

for some $c>0$. In fact, $Q^{*} Q$ has a positive lower bound on the orthogonal complement Ker $Q^{\perp}$ so that

$$
\|u\| \leq C\|Q u\| \quad \text { for } u \in \operatorname{Ker} Q^{\perp}
$$

Thus, if $u=u^{\prime}+u^{\prime \prime}$ with $u^{\prime} \in \operatorname{Ker} Q$ and $u^{\prime \prime} \in \operatorname{Ker} Q^{\perp}$ we find that $Q u=Q u^{\prime \prime}$,

$$
\operatorname{Re}\left\langle(V Q) u^{\prime}, u^{\prime \prime}\right\rangle \geq-\varepsilon\left\|u^{\prime}\right\|^{2}-C_{\varepsilon}\left\|u^{\prime \prime}\right\|^{2} \geq-\varepsilon\left\|u^{\prime}\right\|^{2}-C_{\varepsilon}^{\prime}\|Q u\|^{2} \quad \forall \varepsilon>0
$$

and $\operatorname{Re}\left\langle(V Q) u^{\prime \prime}, u^{\prime \prime}\right\rangle \geq-C\left\|u^{\prime \prime}\right\|^{2} \geq-C^{\prime}\|Q u\|^{2}$. By choosing $\varepsilon$ small enough we obtain (2.8) by using (2.11) on $u^{\prime}$.

Next, we note that $\operatorname{Im} Q^{*}=-\operatorname{Im} Q$ and $\operatorname{Re} Q^{*}=\operatorname{Re} Q$, so $-Q^{*}$ satifies (2.9) and (2.11) with $V$ replaced by $-V$, and thus it is quasi-symmetric. Finally, we shall show that $Q_{E}=E^{*} Q E$ is quasi-symmetric when $E$ is invertible. We obtain from (2.9) that

$$
\operatorname{Im}\left\langle Q_{E} u, u\right\rangle=\operatorname{Im}\langle Q E u, E u\rangle \geq 0 \quad \forall u \in \mathbf{C}^{N}
$$

Next, we shall show that $Q_{E}$ satisfies (2.11) on $\operatorname{Ker} Q_{E}=E^{-1} \operatorname{Ker} Q$, which will give (2.8). We find from Leibniz' rule that $V Q_{E}=\left(V E^{*}\right) Q E+E^{*}(V Q) E+E^{*} Q(V E)$ where (2.11) gives

$$
\operatorname{Re}\left\langle E^{*}(V Q) E u, u\right\rangle \geq c\|E u\|^{2} \geq c^{\prime}\|u\|^{2} \quad u \in \operatorname{Ker} Q_{E} \quad c^{\prime}>0
$$

since then $E u \in \operatorname{Ker} Q$. Similarly we obtain that $\left\langle\left(V E^{*}\right) Q E u, u\right\rangle=0$ when $u \in \operatorname{Ker} Q_{E}$. Now since $\operatorname{Im} Q_{E} \geq 0$ we find from Lemma 2.13 that

$$
\operatorname{Ker} Q_{E}^{*}=\operatorname{Ker} Q_{E}
$$

which gives $\left\langle E^{*} Q(V E) u, u\right\rangle=\left\langle E^{-1}(V E) u, Q_{E}^{*} u\right\rangle=0$ when $u \in \operatorname{Ker} Q_{E}=\operatorname{Ker} Q_{E}^{*}$. Thus $Q_{E}$ satisfies (2.11) so it is quasi-symmetric, which finishes the proof. 
Proposition 2.15. Let $P(w) \in C^{\infty}\left(T^{*} X\right)$ be a quasi-symmetrizable $N \times N$ system, then $P^{*}$ is quasi-symmetrizable. If $A(w)$ and $B(w) \in C^{\infty}\left(T^{*} X\right)$ are invertible $N \times N$ systems then BPA is quasi-symmetrizable.

Proof. Clearly (2.8) -(2.9) are invariant under left multiplication of $P$ with invertible systems $E$, just replace $M$ with $M E^{-1}$. Since we may write $B P A=B\left(A^{*}\right)^{-1} A^{*} P A$ it suffices to show that $E^{*} P E$ is quasi-symmetrizable if $E$ is invertible. By Remark 2.11 there exists a symmetrizer $M$ so that $Q=M P$ is quasi-symmetric, i.e., satisfies $(2.8)-(2.9)$. It then follows from Proposition 2.14 that

$$
Q_{E}=E^{*} Q E=E^{*} M\left(E^{*}\right)^{-1} E^{*} P E
$$

is quasi-symmetric, thus $E^{*} P E$ is quasi-symmetrizable.

Finally, we shall prove that $P^{*}$ is quasi-symmetrizable if $P$ is. Since $Q=M P$ is quasi-symmetric, we find from Proposition 2.14 that $Q^{*}=P^{*} M^{*}$ is quasi-symmetric. By multiplying with $\left(M^{*}\right)^{-1}$ from right, we find from the first part of the proof that $P^{*}$ is quasi-symmetrizable.

For scalar symbols of principal type, we find from the normal form in Example 2.9 that 0 is on the boundary of the local numerical range of the principal symbol. This need not be the case for systems by the following example.

Example 2.16. Let

$$
P(w)=\left(\begin{array}{cc}
w_{2}+i w_{3} & w_{1} \\
w_{1} & w_{2}-i w_{3}
\end{array}\right)
$$

which is quasi-symmetrizable with respect to $\partial_{w_{1}}$ with symmetrizer $M=\left(\begin{array}{ll}0 & 1 \\ 1 & 0\end{array}\right)$. In fact, $\partial_{w_{1}} M P=\mathrm{Id}_{2}$ and

$$
M P(w)=\left(\begin{array}{cc}
w_{1} & w_{2}-i w_{3} \\
w_{2}+i w_{3} & w_{1}
\end{array}\right)=(M P(w))^{*}
$$

so $\operatorname{Im} M P \equiv 0$. Since eigenvalues of $P(w)$ are $w_{2} \pm \sqrt{w_{1}^{2}-w_{3}^{2}}$ we find that 0 is not a boundary point of the local numerical range of the eigenvalues.

For quasi-symmetrizable systems we have the following semiglobal solvability result.

Theorem 2.17. Assume that $P \in \Psi_{c l}^{m}(X)$ is an $N \times N$ system and that there exists a real valued function $T(w) \in C^{\infty}\left(T^{*} X\right)$ such that $P$ is quasi-symmetrizable with respect to the Hamilton vector field $H_{T}(w)$ in a neighborhood of a compactly based cone $K \subset T^{*} X$. Then $P$ is locally solvable at $K$.

The cone $K \subset T^{*} X$ is compactly based if $K \bigcap\{(x, \xi):|\xi|=1\}$ is compact. We also get the following local result: 
Corollary 2.18. Let $P \in \Psi_{c l}^{m}(X)$ be an $N \times N$ system that is is quasi-symmetrizable at $w_{0} \in T^{*} X$. Then $P$ is locally solvable at $w_{0}$.

This follows since we can always choose a function $T$ such that $V=H_{T}$ at $w_{0}$. Recall that a semibicharacteristic of $\lambda \in C^{\infty}$ is a bicharacteristic of $\operatorname{Re}(a \lambda)$ for some $0 \neq a \in C^{\infty}$.

Remark 2.19. If $Q$ is quasi-symmetric with respect to $H_{T}$ then the limit set at the characteristics of the non-trivial semibicharacteristics of the eigenvalues close to zero of $Q$ is a union of curves on which $T$ is strictly monotone, thus they cannot form closed orbits.

In fact, we have that an eigenvalue $\lambda(w)$ is $C^{\infty}$ almost everywhere. The Hamilton vector field $H_{\operatorname{Re} z \lambda}$ then gives the semibicharacteristics of $\lambda$, and that is determined by $\langle d Q u, u\rangle$ with $0 \neq u \in \operatorname{Ker}\left(P-\lambda \operatorname{Id}_{N}\right)$ by the invariance property given by (2.2). Now $\operatorname{Re}\left\langle\left(H_{T} Q\right) u, u\right\rangle>0$ and $\operatorname{Im} d\langle Q u, u\rangle=0$ for $u \in \operatorname{Ker} P$ by (2.8)-(2.9). Thus by picking subsequences when $\lambda \rightarrow 0$ we find that the limits of non-trivial semibicharacteristics of the eigenvalues close to zero give curves on which $T$ is strictly monotone, since $H_{T} \lambda \neq 0$.

Example 2.20. Let

$$
P(t, x ; \tau, \xi)=\tau M(t, x, \xi)+i F(t, x, \xi) \in S_{c l}^{1}
$$

where $M \geq c_{0}>0$ and $F \geq 0$. Then $P$ is quasi-symmetrizable with respect to $\partial_{\tau}$ with symmetrizer $\operatorname{Id}_{N}$, so Theorem 2.17 gives that $P\left(t, x, D_{t}, D_{x}\right)$ is locally solvable.

Proof of Theorem 2.17. We shall modify the proof of Theorem 4.15 in [3], and derive estimates for the $L^{2}$ adjoint $P^{*}$ which will give solvability. By Proposition 2.15 we find that $P^{*}$ is quasi-symmetrizable in $K$. By the invariance of the conditions, we may multiply with an elliptic scalar operator to obtain that $P^{*} \in \Psi_{c l}^{1}$. By the assumptions, Definition 2.8 and (2.7), we find that there exists a real valued function $T(w) \in C^{\infty}$ and a symmetrizer $M(w) \in C^{\infty}$ so that $Q=M P^{*}$ satisfies

$$
\begin{aligned}
& \operatorname{Re} H_{T} Q \geq c-C_{0} Q^{*} Q \geq c-C_{1} \operatorname{Im} Q \\
& \operatorname{Im} Q \geq c Q^{*} Q \geq 0
\end{aligned}
$$

when $|\xi|=1$ near $K$ for some $c>0$, and we find that $M$ is invertible by Remark 2.10. Extending by homogeneity, we may assume that $M$ and $T$ are homogeneous of degree 0 in $\xi$, then $T \in S_{1,0}^{0}$ and $Q \in S_{1,0}^{1}$. Let

$$
M(x, D) P^{*}(x, D)=Q(x, D) \in \Psi_{c l}^{1}
$$

which has principal symbol $Q(x, \xi)$. Leibniz' rule gives that $\exp ( \pm \gamma T) \in S_{1,0}^{0}$ for any $\gamma>0$, so we can define

$$
Q_{\gamma}(x, D)=\exp (-\gamma T)(x, D) Q(x, D) \exp (\gamma T)(x, D) \in \Psi_{c l}^{1}
$$


Since $T$ is a scalar function, we obtain that the symbol of

$$
\operatorname{Im} Q_{\gamma}=Q_{1}+\gamma Q_{0} \quad \text { modulo } S^{-1} \text { near } K
$$

where $0 \leq Q_{1}=\operatorname{Im} Q \in S^{1}$ and $Q_{0} \in S^{0}$ satisfies

$$
Q_{0}=\operatorname{Re} H_{T} Q \geq c-C|\xi|^{-1} Q_{1} \quad \text { near } K
$$

by (2.13), (2.14) and homogeneity.

Now take $0 \leq \phi \in S_{1,0}^{0}$ such that $\phi=1$ near $K$ and $\phi$ is supported where (2.13) and (2.14) hold. If $\chi=\phi^{2}$ then we obtain from (2.17) and the sharp Gårding inequality [5, Theorem 18.6.14] that

$$
Q_{0}(x, D) \geq c_{0} \chi(x, D)-C\langle D\rangle^{-1} Q_{1}(x, D)+R(x, D)+S(x, D)
$$

where $c_{0}>0, R \in S^{-1}$ and $S \in S^{0}$ with $\operatorname{supp} S \bigcap K=\emptyset$. Thus we obtain

$$
\operatorname{Im} Q_{\gamma}(x, D) \geq c_{0} \gamma \chi(x, D)+\left(1+\varrho_{\gamma}\right) Q_{1}(x, D)+R_{\gamma}(x, D)+S_{\gamma}(x, D)
$$

where $R_{\gamma} \in S^{-1}, \varrho_{\gamma}=-\gamma C\langle D\rangle^{-1} \in \Psi^{-1}$ and $S_{\gamma} \in S^{0}$ with $\operatorname{supp} S_{\gamma} \bigcap K=\emptyset$. The calculus gives that $\chi(x, D) \cong \phi(x, D) \phi(x, D)$ modulo $\Psi^{-1}$ and

$$
\left.\left(1+\varrho_{\gamma}\right) Q_{1}(x, D)=\left(1+\varrho_{\gamma} / 2\right)\right) Q_{1}(x, D)\left(1+\varrho_{\gamma} / 2\right) \quad \text { modulo } \Psi^{-1}
$$

By using the sharp Gårding inequality we obtain that $Q_{1}(x, D) \geq R_{0}(x, D)$ for some $R_{0} \in S_{1,0}^{0}$. Thus we find

$$
\left(1+\varrho_{\gamma}\right) Q_{1}(x, D) \geq\left(1+\varrho_{\gamma} / 2\right) R_{0}(x, D)\left(1+\varrho_{\gamma} / 2\right)=R_{0}(x, D) \geq-C_{0}
$$

modulo terms in $\Psi^{-1}$ (depending on $\gamma$ ). Combining this with (2.18) and using that $\operatorname{supp}(1-\phi) \bigcap K=\emptyset$, we find for large enough $\gamma$ that

$$
c_{1} \gamma\|\phi(x, D) u\|^{2} \leq \operatorname{Im}\left\langle Q_{\gamma}(x, D) u, u\right\rangle+\left\langle A_{\gamma}(x, D) u, u\right\rangle+\left\langle B_{\gamma}(x, D) u, u\right\rangle \quad u \in C_{0}^{\infty}
$$

where $c_{1}>0, A_{\gamma} \in S^{-1}$ and $B_{\gamma} \in S^{0}$ with supp $B_{\gamma} \cap K=\emptyset$. Next, we fix $\gamma$ and apply this to $\exp (-\gamma T)(x, D) u$. We find by the calculus that

$$
\|\phi(x, D) u\| \leq C\left(\|\phi(x, D) \exp (-\gamma T)(x, D) u\|+\|u\|_{(-1)}\right) \quad u \in C_{0}^{\infty}
$$

We also obtain from the calculus that

$$
\exp (\gamma T)(x, D) \exp (-\gamma T)(x, D)=1+r(x, D)
$$

with $r \in S^{-1}$, which gives

$$
\begin{aligned}
Q_{\gamma}(x, D) \exp (-\gamma T)(x, D)=\exp (-\gamma T)(x, D)(1 & +r(x, D)) Q(x, D) \\
& +\exp (-\gamma T)(x, D)[Q(x, D), r(x, D)]
\end{aligned}
$$

where $[Q(x, D), r(x, D)] \in \Psi^{-1}$. Since $Q(x, D)=M(x, D) P^{*}(x, D)$ we find

$$
|\langle\exp (-\gamma T)(x, D)(1+r(x, D)) Q(x, D) v, \exp (-\gamma T)(x, D) u\rangle| \leq C\left\|P^{*}(x, D) u\right\|\|u\|
$$


Since $\|u\| \leq\|\phi(x, D) u\|+\|(1-\phi(x, D)) u\|$ and $\phi=1$ near $K$ we obtain that

$$
\|u\| \leq C\left(\left\|P^{*}(x, D) u\right\|+\|Q(x, D) u\|+\|u\|_{(-1)}\right) \quad u \in C_{0}^{\infty}
$$

where $Q \in S^{0}$ with $\operatorname{supp} Q \bigcap K=\emptyset$. We then obtain the local solvability by standard arguments.

\section{Subellipticity of Systems}

We shall consider the question when a quasi-symmetrizable system is subelliptic. Recall that an $N \times N$ system of operators $P \in \Psi_{c l}^{m}(X)$ is (micro)subelliptic with a loss of $\gamma<1$ derivatives at $w_{0}$ if

$$
P u \in H_{(s)} \text { at } w_{0} \Longrightarrow u \in H_{(s+m-\gamma)} \text { at } w_{0}
$$

for $u \in \mathcal{D}^{\prime}\left(X, \mathbf{C}^{N}\right)$. Here $H_{(s)}$ is the standard Sobolev space of distributions $u$ such that $\langle D\rangle^{s} u \in L^{2}$. We say that $u \in H_{(s)}$ microlocally at $w_{0}$ if there exists $a \in S_{1,0}^{0}$ such that $a \neq 0$ in a conical neighborhood of $w_{0}$ and $a(x, D) u \in H_{(s)}$. Of course, ellipticity corresponds to $\gamma=0$ so we shall assume $\gamma>0$.

Example 3.1. Consider the scalar operator

$$
D_{t}+i f\left(t, x, D_{x}\right)
$$

with $0 \leq f \in C^{\infty}\left(\mathbf{R}, S_{c l}^{1}\right),(t, x) \in \mathbf{R} \times \mathbf{R}^{n}$, then we obtain from Proposition 27.3.1 in [5] that this operator is subelliptic with a loss of $k / k+1$ derivatives microlocally near $\{\tau=0\}$ if and only if

$$
\sum_{j \leq k}\left|\partial_{t}^{j} f(t, x, \xi)\right| \neq 0 \quad \forall x \xi
$$

where we can choose $k$ even.

The following example shows that condition (3.1) is not sufficient for systems.

Example 3.2. Let $P=D_{t} \operatorname{Id}_{2}+i F(t)\left|D_{x}\right|$ where

$$
F(t)=\left(\begin{array}{ll}
t^{2} & t^{3} \\
t^{3} & t^{4}
\end{array}\right) \geq 0
$$

Then we have $F^{(3)}(0)=\left(\begin{array}{ll}0 & 6 \\ 6 & 0\end{array}\right)$ which gives that

$$
\bigcap_{j \leq 3} \operatorname{Ker} F^{(j)}(0)=\{0\}
$$

But

$$
F(t)=\left(\begin{array}{cc}
1 & t \\
-t & 1
\end{array}\right)\left(\begin{array}{cc}
t^{2} & 0 \\
0 & 0
\end{array}\right)\left(\begin{array}{cc}
1 & -t \\
t & 1
\end{array}\right)
$$


so we find

$$
P=\left(1+t^{2}\right)^{-1}\left(\begin{array}{cc}
1 & t \\
-t & 1
\end{array}\right)\left(\begin{array}{cc}
D_{t}+i\left(t^{2}+t^{4}\right)\left|D_{x}\right| & 0 \\
0 & D_{t}
\end{array}\right)\left(\begin{array}{cc}
1 & -t \\
t & 1
\end{array}\right) \quad \text { modulo } \Psi^{0}
$$

which is not subelliptic near $\{\tau=0\}$, since $D_{t}$ is not by Example 3.1 .

Example 3.3. Let $P=h D_{t} \operatorname{Id}_{2}+i F(t)\left|D_{x}\right|$ where

$$
F(t)=\left(\begin{array}{cc}
t^{2}+t^{8} & t^{3}-t^{7} \\
t^{3}-t^{7} & t^{4}+t^{6}
\end{array}\right)=\left(\begin{array}{cc}
1 & t \\
-t & 1
\end{array}\right)\left(\begin{array}{cc}
t^{2} & 0 \\
0 & t^{6}
\end{array}\right)\left(\begin{array}{cc}
1 & -t \\
t & 1
\end{array}\right)
$$

Then we have

$$
P=\left(1+t^{2}\right)^{-1}\left(\begin{array}{cc}
1 & t \\
-t & 1
\end{array}\right)\left(\begin{array}{cc}
D_{t}+i\left(t^{2}+t^{4}\right)\left|D_{x}\right| & 0 \\
0 & D_{t}+i\left(t^{6}+t^{8}\right)\left|D_{x}\right|
\end{array}\right)\left(\begin{array}{cc}
1 & -t \\
t & 1
\end{array}\right)
$$

modulo $\Psi^{0}$, which is subelliptic near $\{\tau=0\}$ with a loss of $6 / 7$ derivatives by Example 3.1. This operator is, element for element, a higher order perturbation of the operator of Example 3.2 .

The problem is that condition (3.2) in not invariant in the systems case. Instead, we shall consider the following invariant generalization of (3.1).

Definition 3.4. Let $0 \leq F(t) \in L_{l o c}^{\infty}(\mathbf{R})$ be an $N \times N$ system, then we define

$$
\Omega_{\delta}(F)=\left\{t: \min _{\|u\|=1}\langle F(t) u, u\rangle \leq \delta\right\} \quad \delta>0
$$

which is well-defined almost everywhere and contains $|F|^{-1}(0)$.

Observe that one may also use this definition in the scalar case, then $\Omega_{\delta}(f)=f^{-1}([0, \delta])$ for non-negative functions $f$.

Remark 3.5. Observe that if $F \geq 0$ and $E$ is invertible then we find that

$$
\Omega_{\delta}\left(E^{*} F E\right) \subseteq \Omega_{C \delta}(F)
$$

where $C=\left\|E^{-1}\right\|^{2}$.

Example 3.6. For the matrix $F(t)$ in Example 3.3 we find that $\left|\Omega_{\delta}(F)\right| \leq C \delta^{1 / 6}$ for $0<\delta \leq 1$, and for the matrix in Example 3.2 we find that $\left|\Omega_{\delta}(F)\right|=\infty, \forall \delta$.

We also have examples when the semidefinite imaginary part vanishes of infinite order.

Example 3.7. Let $0 \leq f(t, x) \leq C e^{-1 /|t|^{\sigma}}, \sigma>0$, then we obtain that

$$
\left|\Omega_{\delta}\left(f_{x}\right)\right| \leq C_{0}|\log \delta|^{-1 / \sigma} \quad \forall \delta>0 \quad \forall x
$$

where $f_{x}(t)=f(t, x)$. (We owe this example to Y. Morimoto.) 
We shall study systems where the imaginary part $F$ vanishes of finite order, so that $\left|\Omega_{\delta}(F)\right| \leq C \delta^{\mu}$ for $\mu>0$. In general, the largest exponent could be any $\mu>0$, for example when $F(t)=|t|^{1 / \mu} \operatorname{Id}_{N}$. But for $C^{\infty}$ systems the best exponent is $\mu=1 / k$ for an even $k$, by the following result, which is Proposition A.2 in [3].

Remark 3.8. Assume that $0 \leq F(t) \in C^{\infty}(\mathbf{R})$ is an $N \times N$ system such that $F(t) \geq c>0$ when $|t| \gg 1$. Then we find that

$$
\left|\Omega_{\delta}(F)\right| \leq C \delta^{\mu} \quad 0<\delta \leq 1
$$

if and only if $\mu \leq 1 / k$ for an even $k \geq 0$ so that

$$
\sum_{j \leq k}\left|\partial_{t}^{j}\langle F(t) u(t), u(t)\rangle\right| /\|u(t)\|^{2}>0 \quad \forall t
$$

for any $0 \neq u(t) \in C^{\infty}(\mathbf{R})$.

Example 3.9. For the scalar symbols $\tau+i f(t, x, \xi)$ in Example 3.1 we find from Remark 3.8 that (3.1) is equivalent to

$$
|\{t: f(t, x, \xi) \leq \delta\}|=\left|\Omega_{\delta}\left(f_{x, \xi}\right)\right| \leq C \delta^{1 / k} \quad 0<\delta \leq 1 \quad|\xi|=1
$$

where $f_{x, \xi}(t)=f(t, x, \xi)$.

The following example shows that for subelliptic type of estimates it is not sufficient to have conditions only on the vanishing of the symbol, we also need conditions on the semibicharacteristics of the eigenvalues.

Example 3.10. Let

$$
P=D_{t} \operatorname{Id}_{2}+\alpha\left(\begin{array}{cc}
D_{x} & 0 \\
0 & -D_{x}
\end{array}\right)+i(t-\beta x)^{2}\left|D_{x}\right| \operatorname{Id}_{2} \quad(t, x) \in \mathbf{R}^{2}
$$

with $\alpha, \beta \in \mathbf{R}$, then we see from the scalar case in Example 3.1 that $P$ is subelliptic near $\{\tau=0\}$ with a loss of $2 / 3$ derivatives if and only either $\alpha=0$ or $\alpha \neq 0$ and $\beta \neq \pm 1 / \alpha$.

Definition 3.11. Let $Q \in C^{\infty}\left(T^{*} X\right)$ be an $N \times N$ system and let $w_{0} \in \Sigma \subset T^{*} X$, then $Q$ satisfies the approximation property on $\Sigma$ near $w_{0}$ if there exists a $Q$ invariant $C^{\infty}$ subbundle $\mathcal{V}$ of $\mathbf{C}^{N}$ over $T^{*} X$ such that $\mathcal{V}\left(w_{0}\right)=\operatorname{Ker} Q^{N}\left(w_{0}\right)$ and

$$
\operatorname{Re}\langle Q(w) v, v\rangle=0 \quad v \in \mathcal{V}(w) \quad w \in \Sigma
$$

near $w_{0}$. That $\mathcal{V}$ is $Q$ invariant means that $Q(w) v \in \mathcal{V}(w)$ for $v \in \mathcal{V}(w)$.

Here $\operatorname{Ker} Q^{N}\left(w_{0}\right)$ is the space of the generalized eigenvectors corresponding to the zero eigenvalue. The symbol of the system in Example 3.10 satisfies the approximation property on $\Sigma=\{\tau=0\}$ if and only if $\alpha=0$. 
Let $\widetilde{Q}=\left.Q\right|_{\mathcal{V}}$ then $\operatorname{Im} i \widetilde{Q}=\operatorname{Re} \widetilde{Q}=0$ so Lemma 2.13 gives that $\operatorname{Ran} \widetilde{Q} \perp \operatorname{Ker} \widetilde{Q}$ on $\Sigma$. Thus $\operatorname{Ker} \widetilde{Q}^{N}=\operatorname{Ker} \widetilde{Q}$ on $\Sigma$, and since $\operatorname{Ker} \widetilde{Q}^{N}\left(w_{0}\right)=\mathcal{V}\left(w_{0}\right)$ we find that $\operatorname{Ker} Q^{N}\left(w_{0}\right)=$ $\mathcal{V}\left(w_{0}\right)=\operatorname{Ker} Q\left(w_{0}\right)$.

Remark 3.12. Assume that $Q$ satisfies the approximation property on the $C^{\infty}$ hypersurface $\Sigma$ and is quasi-symmetric with respect to $V \notin T \Sigma$. Then the limits of the non-trivial semibicharacteristics of the eigenvalues of $Q$ close to zero coincide with the bicharacteristics of $\Sigma$.

In fact, the approximation property in Definition 3.11 gives that $\langle\operatorname{Re} Q u, u\rangle=0$ for $u \in \operatorname{Ker} Q$ when $\tau=0$. Since $\operatorname{Im} Q \geq 0$ we find that

$$
\langle d Q u, u\rangle=0 \quad \forall u \in \operatorname{Ker} Q \quad \text { on } T \Sigma
$$

By Remark 2.19 the limits of the non-trivial semibicharacteristics of the eigenvalues close to zero of $Q$ are curves with tangents determined by $\langle d Q u, u\rangle$ for $u \in \operatorname{Ker} Q$. Since $V \operatorname{Re} Q \neq 0$ on $\operatorname{Ker} Q$ we find from (3.7) that the limit curves coincide with the bicharacteristics of $\Sigma$, which are the flow-outs of the Hamilton vector field.

Example 3.13. Observe that Definition 3.11 is empty if $\operatorname{Dim} \operatorname{Ker} Q^{N}\left(w_{0}\right)=0$. If $\operatorname{Dim} \operatorname{Ker} Q^{N}\left(w_{0}\right)>0$, then there exists $\varepsilon>0$ and a neigborhood $\omega$ to $w_{0}$ so that

$$
\Pi(w)=\frac{1}{2 \pi i} \int_{|z|=\varepsilon}\left(z \operatorname{Id}_{N}-Q(w)\right)^{-1} d z \in C^{\infty}(\omega)
$$

is the spectral projection on the (generalized) eigenvectors with eigenvalues having absolute value less than $\varepsilon$. Then $\operatorname{Ran} \Pi$ is a $Q$ invariant bundle over $\omega$ so that $\operatorname{Ran} \Pi\left(w_{0}\right)=$ $\operatorname{Ker} Q^{N}\left(w_{0}\right)$. Condition (3.6) with $\mathcal{V}=\operatorname{Ran} \Pi$ means that $\Pi^{*} \operatorname{Re} Q \Pi \equiv 0$ in $\omega$. When $\operatorname{Im} Q\left(w_{0}\right) \geq 0$ we find that $\Pi^{*} Q \Pi\left(w_{0}\right)=0$, then $Q$ satisfies the approximation property on $\Sigma$ near $w_{0}$ with $\mathcal{V}=\operatorname{Ran} \Pi$ if and only if

$$
\left.d\left(\Pi^{*}(\operatorname{Re} Q) \Pi\right)\right|_{T \Sigma} \equiv 0 \quad \text { near } w_{0}
$$

Example 3.14. If $Q$ satisfies the approximation property on $\Sigma$, then by choosing an orthonormal basis for $\mathcal{V}$ and extending it to an orthonormal basis for $\mathbf{C}^{N}$ we obtain the system on the form

$$
Q=\left(\begin{array}{cc}
Q_{11} & Q_{12} \\
0 & Q_{22}
\end{array}\right)
$$

where $Q_{11}$ is $K \times K$ system such that $Q_{11}^{N}\left(w_{0}\right)=0, \operatorname{Re} Q_{11}=0$ on $\Sigma$ and $\left|Q_{22}\right| \neq 0$. By multiplying from left with

$$
\left(\begin{array}{cc}
\operatorname{Id}_{K} & -Q_{12} Q_{22}^{-1} \\
0 & \operatorname{Id}_{N-K}
\end{array}\right)
$$

we obtain that $Q_{12} \equiv 0$ without changing $Q_{11}$ or $Q_{22}$. 
In fact, the eigenvalues of $Q$ are then eigenvalues of either $Q_{11}$ or $Q_{22}$. Since $\mathcal{V}\left(w_{0}\right)$ are the (generalized) eigenvectors corresponding to the zero eigenvalue of $Q\left(w_{0}\right)$ we find that all eigenvalues of $Q_{22}\left(w_{0}\right)$ are non-vanishing, thus $Q_{22}$ is invertible near $w_{0}$,

Remark 3.15. If $Q$ satisfies the approximation property on $\Sigma$ near $w_{0}$, then it satisfies the approximation property on $\Sigma$ near $w_{1}$, for $w_{1}$ sufficiently close to $w_{0}$.

In fact, let $Q_{11}$ be the restriction of $Q$ to $\mathcal{V}$ as in Example 3.14, then since $\operatorname{Re} Q_{11}=$ $\operatorname{Im} i Q_{11}=0$ on $\Sigma$ we find from Lemma 2.13 that $\operatorname{Ran} Q_{11} \perp \operatorname{Ker} Q_{11}$ and $\operatorname{Ker} Q_{11}=\operatorname{Ker} Q_{11}^{N}$ on $\Sigma$. Since $Q_{22}$ is invertible in (3.14), we find that $\operatorname{Ker} Q \subseteq \mathcal{V}$. Thus, by using the spectral projection (3.8) of $Q_{11}$ near $w_{1} \in \Sigma$ for small enough $\varepsilon$ we obtain an $Q$ invariant subbundle $\widetilde{\mathcal{V}} \subseteq \mathcal{V}$ so that $\widetilde{\mathcal{V}}\left(w_{1}\right)=\operatorname{Ker} Q_{11}\left(w_{1}\right)=\operatorname{Ker} Q^{N}\left(w_{1}\right)$

If $Q \in C^{\infty}$ satisfies the approximation property and $Q_{E}=E^{*} Q E$ with invertible $E \in C^{\infty}$, then it follows from the proof of Proposition 3.20 below that there exist invertible $A, B \in C^{\infty}$ so that $A Q_{E}$ and $Q^{*} B$ satisfy the approximation property.

Definition 3.16. Let $P(w) \in C^{\infty}\left(T^{*} X\right)$ be an $N \times N$ system and $\mu \in \mathbf{R}_{+}$. Then $P$ is of finite type $\mu$ at $w_{0} \in T^{*} X$ if there exists a neighborhood $\omega$ of $w_{0}$, a $C^{\infty}$ hypersurface $\Sigma \ni$ $w_{0}$, a real $C^{\infty}$ vector field $V \notin T \Sigma$ and an invertible symmetrizer $M \in C^{\infty}$ so that $Q=M P$ is quasi-symmetric with respect to $V$ in $\omega$ and satisfies the approximation property on $\Sigma \bigcap \omega$. Also, for every bicharacteristic $\gamma$ of $\Sigma$ the arc length

$$
\left|\gamma \cap \Omega_{\delta}(\operatorname{Im} Q) \cap \omega\right| \leq C \delta^{\mu} \quad 0<\delta \leq 1
$$

The operator $P \in \Psi_{c l}^{m}$ is of finite type $\mu$ at $w_{0}$ if the principal symbol $\sigma(P)$ is of finite type when $|\xi|=1$.

Recall that the bicharacteristics of a hypersurface in $T^{*} X$ are the flow-outs of the Hamilton vector field of $\Sigma$. Of course, if $P$ is elliptic then it is trivially of finite type 0 , just choose $M=i P^{-1}$ to obtain $Q=i \operatorname{Id}_{N}$. If $P$ is of finite type, then it is quasisymmetrizable by definition and thus of principal type.

Remark 3.17. Observe that since $0 \leq \operatorname{Im} Q \in C^{\infty}$ we obtain from Remark 3.8 that the largest exponent in (3.9) is $\mu=1 / k$ for an even $k \geq 0$. Also, we may assume that

$$
\operatorname{Im}\langle Q u, u\rangle \geq c\|Q u\|^{2} \quad \forall u \in \mathbf{C}^{N}
$$

In fact, by adding $i \varrho P^{*}$ to $M$ we obtain (3.10) for large enough $\varrho$ by (2.7), and this does not change $\operatorname{Re} Q$.

Example 3.18. Assume that $Q$ is quasi-symmetric with respect to the real vector field $V$, satisfying (3.9) and the approximation property on $\Sigma$. Then by choosing an orthonormal 
basis and changing the symmetrizer as in Example 3.14 we obtain the system on the form

$$
Q=\left(\begin{array}{cc}
Q_{11} & 0 \\
0 & Q_{22}
\end{array}\right)
$$

where $Q_{11}$ is $K \times K$ system such that $Q_{11}^{N}\left(w_{0}\right)=0$, $\operatorname{Re} Q_{11}=0$ on $\Sigma$ and $\left|Q_{22}\right| \neq 0$. Since $Q$ is quasi-symmetric with respect to $V$ we also obtain that $Q_{11}\left(w_{0}\right)=0, \operatorname{Re} V Q_{11}>0$, $\operatorname{Im} Q \geq 0$ and $Q$ satisfies (3.9). In fact, then we find from Lemma 2.13 that $\operatorname{Im} Q \perp \operatorname{Ker} Q$ which gives $\operatorname{Ker} Q^{N}=\operatorname{Ker} Q$. Note that $\Omega_{\delta}\left(\operatorname{Im} Q_{11}\right) \subseteq \Omega_{\delta}(\operatorname{Im} Q)$, so $Q_{11}$ satisfies (3.9)).

Example 3.19. In the scalar case, we find from Example 2.9 that $p \in C^{\infty}\left(T^{*} X\right)$ is quasi-symmetrizable with respect to $H_{t}=\partial_{\tau}$ if and only if

$$
p(t, x ; \tau, \xi)=q(t, x ; \tau, \xi)(\tau+i f(t, x, \xi))
$$

with $f \geq 0$ and $q \neq 0$. If $f(t, x, \xi) \geq c>0$ when $|(t, x, \xi)| \gg 1$ we find by taking $q^{-1}$ as symmetrizer that $p$ is of finite type $\mu$ if and only if $\mu=1 / k$ for an even $k$ such that

$$
\sum_{j \leq k}\left|\partial_{t}^{k} f(t, x, \xi)\right|>0 \quad \forall x \xi
$$

by Remark 3.8. In fact, the approximation property on $\Sigma=\{\tau=0\}$ is trivial since $f$ is real.

Proposition 3.20. If $P(w) \in C^{\infty}\left(T^{*} X\right)$ is of finite type $\mu$ at $w$ then $P^{*}$ is of finite type $\mu$ at $w$. If $A(w)$ and $B(w) \in C^{\infty}\left(T^{*} X\right)$ are invertible, then $A P B$ is of finite type $\mu$ at $w$.

Proof. Let $M$ be the symmetrizer in Definition 3.16 so that $Q=M P$ is quasi-symmetric with respect to $V$. By choosing a suitable basis and changing the symmetrizer as in Example 3.18, we may write

$$
Q=\left(\begin{array}{cc}
Q_{11} & 0 \\
0 & Q_{22}
\end{array}\right)
$$

where $Q_{11}$ is $K \times K$ system such that $Q_{11}\left(w_{0}\right)=0, V \operatorname{Re} Q_{11}>0, \operatorname{Re} Q_{11}=0$ on $\Sigma$ and $Q_{22}$ is invertible. We also have $\operatorname{Im} Q \geq 0$ and $Q$ satisfies (3.9). Let $\mathcal{V}_{1}=$ $\left\{u \in \mathbf{C}^{N}: u_{j}=0\right.$ for $\left.j>K\right\}$ and $\mathcal{V}_{2}=\left\{u \in \mathbf{C}^{N}: u_{j}=0\right.$ for $\left.j \leq K\right\}$, these are $Q$ invariant bundles such that $\mathcal{V}_{1} \oplus \mathcal{V}_{2}=\mathbf{C}^{N}$.

First we are going to show that $\widetilde{P}=A P B$ is of finite type. By taking $\widetilde{M}=B^{-1} M A^{-1}$ we find that

$$
\widetilde{M} \widetilde{P}=\widetilde{Q}=B^{-1} Q B
$$

and it is clear that $B^{-1} \mathcal{V}_{j}$ are $\widetilde{Q}$ invariant bundles, $j=1,2$. By choosing bases in $B^{-1} \mathcal{V}_{j}$ for $j=1,2$, we obtain a basis for $\mathbf{C}^{N}$ in which $\widetilde{Q}$ has a block form:

$$
\widetilde{Q}=\left(\begin{array}{cc}
\widetilde{Q}_{11} & 0 \\
0 & \widetilde{Q}_{22}
\end{array}\right)
$$


Here $\widetilde{Q}_{j j}: B^{-1} \mathcal{V}_{j} \mapsto B^{-1} \mathcal{V}_{j}$, is given by $\widetilde{Q}_{j j}=B_{j}^{-1} Q_{j j} B_{j}$ with

$$
B_{j}: B^{-1} \mathcal{V}_{j} \ni u \mapsto B u \in \mathcal{V}_{j} \quad j=1,2
$$

By multiplying $\widetilde{Q}$ from the left with

$$
\mathcal{B}=\left(\begin{array}{cc}
B_{1}^{*} B_{1} & 0 \\
0 & B_{2}^{*} B_{2}
\end{array}\right)
$$

we obtain that

$$
\bar{Q}=\mathcal{B} \widetilde{Q}=\mathcal{B} \widetilde{M} \widetilde{P}=\left(\begin{array}{cc}
B_{1}^{*} Q_{11} B_{1} & 0 \\
0 & B_{2}^{*} Q_{22} B_{2}
\end{array}\right)=\left(\begin{array}{cc}
\bar{Q}_{11} & 0 \\
0 & \bar{Q}_{22}
\end{array}\right)
$$

It is clear that $\operatorname{Im} \bar{Q} \geq 0, Q_{11}\left(w_{0}\right)=0, \operatorname{Re} \bar{Q}_{11}=0$ on $\Sigma,\left|\bar{Q}_{22}\right| \neq 0$ and $V \operatorname{Re} \bar{Q}_{11}>0$ by Proposition 2.14, Finally, we obtain from Remark 3.5 that

$$
\Omega_{\delta}(\operatorname{Im} \bar{Q}) \subseteq \Omega_{C \delta}(\operatorname{Im} Q)
$$

for some $C>0$, which proves that $\widetilde{P}=A P B$ is of finite type. Observe that $\bar{Q}=A Q_{B}$, where $Q_{B}=B^{*} Q B$ and $A=\mathcal{B} B^{-1}\left(B^{*}\right)^{-1}$.

To show that $P^{*}$ also is of finite type, we may assume as before that $Q=M P$ is on the form (3.12) with $Q_{11}\left(w_{0}\right)=0, V \operatorname{Re} Q_{11}>0, \operatorname{Re} Q_{11}=0$ on $\Sigma, Q_{22}$ is invertible, $\operatorname{Im} Q \geq 0$ and $Q$ satisfies (3.9). Then we find that

$$
-P^{*} M^{*}=-Q^{*}=\left(\begin{array}{cc}
-Q_{11}^{*} & 0 \\
0 & -Q_{22}^{*}
\end{array}\right)
$$

satisfies the same conditions with respect to $-V$, so it is of finite type with multiplier $\operatorname{Id}_{N}$. By the first part of the proof we obtain that $P^{*}$ is of finite type, which finishes the proof.

Theorem 3.21. Assume that $P \in \Psi_{c l}^{m}(X)$ is an $N \times N$ system of finite type $\mu>0$ near $w_{0} \in T^{*} X \backslash 0$, then $P$ is subelliptic at $w_{0}$ with a loss of $1 / \mu+1$ derivatives:

$$
P u \in H_{(s)} \text { at } w_{0} \Longrightarrow u \in H_{(s+m-1 / \mu+1)} \text { at } w_{0}
$$

for $u \in \mathcal{D}^{\prime}\left(X, \mathbf{C}^{N}\right)$.

Observe that the largest exponent is $\mu=1 / k$ for an even $k$ by Remark 3.17, and then $1 / \mu+1=k / k+1$. Thus Theorem 3.21 generalizes Proposition 27.3.1 in [5] by Example 3.19 .

Example 3.22. Let

$$
P(t, x ; \tau, \xi)=\tau M(t, x, \xi)+i F(t, x, \xi) \in S_{c l}^{1}
$$

where $M \geq c_{0}>0$ and $F \geq 0$ satisfies

$$
\left|\left\{t: \inf _{|u|=1}\langle F(t, x, \xi) u, u\rangle \leq \delta\right\}\right| \leq C \delta^{\mu} \quad|\xi|=1
$$


for some $\mu>0$. Then $P$ is quasi-symmetrizable with respect to $\partial_{\tau}$ with symmetrizer $\operatorname{Id}_{N}$. When $\tau=0$ we obtain that $\operatorname{Re} P=0$, so by taking $\mathcal{V}=\operatorname{Ran} \Pi$ for the spectral projection $\Pi$ given by (3.8) for $F$, we find that $P$ satisfies the approximation property with respect to $\Sigma=\{\tau=0\}$. Since $\Omega_{\delta}(\operatorname{Im} P)=\Omega_{\delta}(F)$ we find from (3.17) that $P$ is of finite type $\mu$. Observe that if $F(t, x, \xi) \geq c>0$ when $|(t, x, \xi)| \gg 1$ we find from Remark 3.8 that (3.17) is satisfied if and only if $\mu \leq 1 / k$ for an even $k \geq 0$ so that

$$
\sum_{j \leq k}\left|\partial_{t}^{j}\langle F(t, x, \xi) u(t), u(t)\rangle\right|>0 \quad \forall t, x, \xi
$$

for any $0 \neq u(t) \in C^{\infty}(\mathbf{R})$. Theorem 3.21 gives that $P\left(t, x, D_{t}, D_{x}\right)$ is subelliptic near $\{\tau=0\}$ with a loss of $k / k+1$ derivatives.

Proof of Theorem 3.21. First, we may reduce to the case $m=s=0$ by replacing $u$ and $P$ by $\langle D\rangle^{s+m} u$ and $\langle D\rangle^{s} P\langle D\rangle^{-s-m} \in \Psi_{c l}^{0}$. Now $u \in H_{(-K)}$ for some $K$ near $w_{0}$, and it is no restriction to assume $K=1$. In fact, if $K>1$ then by using that $P u \in H_{(1-K)}$ near $w_{0}$, we obtain that $u \in H_{(-K+\mu / \mu+1)}$ near $w_{0}$ and we may iterate this argument until $u \in H_{(-1)}$ near $w_{0}$. By cutting off with $\phi \in S_{1,0}^{0}$ we may assume that $v=\phi(x, D) u \in H_{(-1)}$ and $P v=[P, \phi(x, D)] u+\phi(x, D) P u \in H_{(0)}$ since $[P, \phi(x, D)] \in \Psi^{-1}$. If $\phi \neq 0$ in a conical neighborhood of $w_{0}$ it suffices to prove that $v \in H_{(-1 / \mu+1)}$.

By Definition 3.16 and Remark 3.17 there exist a $C^{\infty}$ hypersurface $\Sigma$, a real $C^{\infty}$ vector field $V \notin T \Sigma$, an invertible symmetrizer $M \in C^{\infty}$ so that $Q=M P$ satisfies (3.9), the approximation property on $\Sigma$, and

$$
\begin{aligned}
& V \operatorname{Re} Q \geq c-C \operatorname{Im} Q \quad c>0 \\
& \operatorname{Im} Q \geq c Q^{*} Q
\end{aligned}
$$

in a neighborhood $\omega$ of $w_{0}$. By extending by homogeneity, we can assume that $V, M$ and $Q$ are homogeneous of degree 0 .

Since (3.18) is stable under small perturbations in $V$ we can replace $V$ with $H_{t}$ for some real $t \in C^{\infty}$. By solving the initial value problem $H_{t} \tau \equiv-1,\left.\tau\right|_{\Sigma}=0$, and completing to a symplectic $C^{\infty}$ coordinate system $(t, \tau, x, \xi)$, we obtain that $\Sigma=\{\tau=0\}$ in a neighborhood of $w_{0}=\left(0,0, x_{0}, \xi_{0}\right), \xi_{0} \neq 0$. We obtain from Definition 3.11 that

$$
\operatorname{Re}\langle Q u, u\rangle=0 \quad \text { when } u \in \mathcal{V} \text { and } \tau=0
$$

near $w_{0}$. Here $\mathcal{V}$ is a $Q$ invariant $C^{\infty}$ subbundle of $\mathbf{C}^{N}$ such that $\mathcal{V}\left(w_{0}\right)=\operatorname{Ker} Q^{N}\left(w_{0}\right)=$ $\operatorname{Ker} Q\left(w_{0}\right)$ by Lemma 2.13, By condition (3.9) we have that

$$
\left|\Omega_{\delta}\left(\operatorname{Im} Q_{x, \xi}\right) \cap\{|t|<c\}\right| \leq C \delta^{\mu}
$$

when $\left|(x, \xi)-\left(x_{0}, \xi_{0}\right)\right|<c$, here $Q_{x, \xi}(t)=Q(t, 0, x, \xi)$. 
Next, we shall localize the estimate. Choose $\left\{\varphi_{j}\right\}_{j} \in S_{1,0}^{0}$ and $\left\{\psi_{j}\right\}_{j} \in S_{1,0}^{0}$ with values in $\ell^{2}$, such that $\varphi_{j} \geq 0, \psi_{j} \geq 0, \sum_{j} \varphi_{j}^{2}=1, \psi_{j} \varphi_{j} \equiv \varphi_{j}$ and $\psi_{j}$ is supported where $|(\tau, \xi)| \cong 2^{j}$. Since these are Fourier multipliers we find that $\sum_{j} \varphi_{j}\left(D_{t, x}\right)^{2}=1$ and

$$
\|u\|_{(s)}^{2} \cong \sum_{j} 2^{2 s j}\left\|\varphi_{j}\left(D_{t, x}\right) u\right\|^{2} \quad u \in \mathcal{S}
$$

Let $Q_{j}=\psi_{j} Q$ be the localized symbol, and let $h_{j}=2^{-j} \leq 1$. Since $Q_{j} \in S_{1,0}^{0}$ is supported where $|(\tau, \xi)| \cong 2^{j}$, we find that $Q_{j}(t, x, \tau, \xi)=\widetilde{Q}_{j}\left(t, x, h_{j} \tau, h_{j} \xi\right)$ where $\widetilde{Q}_{j} \in$ $C_{0}^{\infty}\left(T^{*} \mathbf{R}^{n}\right)$ uniformly. We shall obtain Theorem 3.21 from the following result, which is Proposition 6.1 in $[3]$.

Proposition 3.23. Assume that $Q \in C_{\mathrm{b}}^{\infty}\left(T^{*} \mathbf{R}^{n}\right)$ is an $N \times N$ system satisfying (3.18)(3.21) in a neighborhood of $w_{0}=\left(0,0, x_{0}, \xi_{0}\right)$ with $V=\partial_{\tau}$ and $\mu>0$. Then there exists $h_{0}>0$ and $R \in C_{\mathrm{b}}^{\infty}\left(T^{*} \mathbf{R}^{n}\right)$ so that $w_{0} \notin \operatorname{supp} R$ and

$$
h^{1 / \mu+1}\|u\| \leq C\left(\left\|Q\left(t, x, h D_{t, x}\right) u\right\|+\left\|R^{w}\left(t, x, h D_{t, x}\right) u\right\|+h\|u\|\right) \quad 0<h \leq h_{0}
$$

for any $u \in C_{0}^{\infty}\left(\mathbf{R}^{n}, \mathbf{C}^{N}\right)$.

Here $C_{\mathrm{b}}^{\infty}$ are $C^{\infty}$ functions with $L^{\infty}$ bounds on any derivative, and the result is uniform in the usual sense. Observe that this estimate can be extended to a semiglobal estimate. In fact, let $\omega$ be a neighborhood of $w_{0}$ such that $\operatorname{supp} R \bigcap \omega=\emptyset$, where $R$ is given by Proposition 3.23. Take $\varphi \in C_{0}^{\infty}(\omega)$ such that $0 \leq \varphi \leq 1$ and $\varphi=1$ in a neighborhood of $w_{0}$. By substituting $\varphi\left(t, x, h D_{t, x}\right) u$ in (3.22) we obtain from the calculus

$$
h^{1 / \mu+1}\left\|\varphi\left(t, x, h D_{t, x}\right) u\right\| \leq C_{N}\left(\left\|\varphi\left(t, x, h D_{t, x}\right) Q\left(t, x, h D_{t, x}\right) u\right\|+h\|u\|\right) \quad \forall u \in C_{0}^{\infty}
$$

for small enough $h$ since $R \varphi \equiv 0$ and $\left\|\left[Q\left(t, x, h D_{t, x}\right), \varphi\left(t, x, h D_{t, x}\right)\right] u\right\| \leq C h\|u\|$. Thus, if $Q$ satisfies conditions (3.18)-(3.21) near any $w \in K \Subset T^{*} \mathbf{R}^{n}$, then by using BolzanoWeierstrass we obtain the estimate (3.22) with $\operatorname{supp} R \cap K=\emptyset$.

Now, by using that $\widetilde{Q}_{j}$ satisfies (3.18)-(3.21) in a neighborhood of $\operatorname{supp} \varphi_{j}$, we obtain the estimate (3.22) for $\widetilde{Q}_{j}\left(t, x, h D_{t, x}\right)$ with $h=h_{j}=2^{-j} \ll 1$ and $R=R_{j} \in S_{1,0}^{0}$ such that $\operatorname{supp} \varphi_{j} \bigcap \operatorname{supp} R_{j}=\emptyset$. Substituting $\varphi_{j}\left(D_{t, x}\right) u$ we obtain for $j \gg 1$ that

$2^{-j / \mu+1}\left\|\varphi_{j}\left(D_{t, x}\right) u\right\| \leq C_{N}\left(\left\|Q_{j}\left(t, x, D_{t, x}\right) \varphi_{j}\left(D_{t, x}\right) u\right\|+\left\|\widetilde{R}_{j} u\right\|+2^{-j}\left\|\varphi_{j}\left(D_{t, x}\right) u\right\|\right) \quad \forall u \in \mathcal{S}^{\prime}$

where $\widetilde{R}_{j}=R_{j}\left(t, x, D_{t, x}\right) \varphi_{j}\left(D_{t, x}\right) \in \Psi^{-N}$ with values in $\ell^{2}$. Now since $Q_{j}$ and $Q$ are uniformly bounded in $S_{1,0}^{0}$ the calculus gives that

$$
Q_{j}\left(t, x, D_{t, x}\right) \varphi_{j}\left(D_{t, x}\right)=\varphi_{j}\left(D_{t, x}\right) Q\left(t, x, D_{t, x}\right)+\varrho_{j}\left(t, x, D_{t, x}\right)
$$


where $\left\{\varrho_{j}\right\}_{j} \in \Psi^{-1}$ with values in $\ell^{2}$. Thus, by squaring and summing up, we obtain by continuity that

$$
\|u\|_{(-1 / \mu+1)}^{2} \leq C\left(\left\|Q\left(t, x, D_{t, x}\right) u\right\|^{2}+\|u\|_{(-1)}^{2}\right) \quad u \in H_{(-1)}
$$

Since $Q\left(t, x, D_{t, x}\right)=M\left(t, x, D_{t, x}\right) P\left(t, x, D_{t, x}\right)$ modulo $\Psi^{-1}$ where $M \in \Psi^{0}$, the calculus gives

$$
\begin{aligned}
\left\|Q\left(t, x, D_{t, x}\right) u\right\| \leq C\left(\| M\left(t, x, D_{t, x}\right)\right. & \left.P\left(t, x, D_{t, x}\right) u\|+\| u \|_{(-1)}\right) \\
& \leq C^{\prime}\left(\left\|P\left(t, x, D_{t, x}\right) u\right\|+\|u\|_{(-1)}\right) \quad u \in H_{(-1)}
\end{aligned}
$$

which together with (3.24) proves Theorem 3.21,

\section{REFERENCES}

1. N. Dencker, J. Sjöstrand, and M. Zworski, Pseudospectra of semiclassical (pseudo-) differential operators, Comm. Pure Appl. Math. 57 (2004), no. 3, 384-415.

2. N. Dencker, The resolution of the Nirenberg-Treves conjecture, Ann. of Math. 163 (2006), 405-444.

3. _ The pseudospectrum of systems of semiclassical operators, arXiv:0705.4561 [math.AP]. To appear in Analysis \& PDE.

4. _ On the solvability of systems of pseudodifferential operators, arXiv:0801.4043 [math.AP]. To appear in Geometric Aspects of Analysis and Mechanics, A Conference in Honor of Hans Duistermaat.

5. L. Hörmander, The analysis of linear partial differential operators, vol. I-IV, Springer-Verlag, Berlin, 1983-1985.

6. H. Lewy An example of a smooth linear partial differential equation without solution, Ann. of Math. 66 (1957), 155-158.

7. L. Nirenberg and F. Treves, On local solvability of linear partial differential equations. Part I: Necessary conditions, Comm. Partial Differential Equations 23 (1970), 1-38, Part II: Sufficient conditions, Comm. Pure Appl. Math. 23 (1970), 459-509; Correction, Comm. Pure Appl. Math. 24 (1971), 279-288.

8. M. Taylor, Pseudodifferential operators, Princeton University Press, Princeton, N.J., 1981.

Centre for Mathematical Sciences, Lund University, Box 118, SE-221 00 Lund, Sweden

E-mail address: dencker@maths.1th.se 